\title{
Modelo MIMO Nebuloso com Estrutura Mamdani Aplicado à Análise e Controle de Vazão Hídrica
}

\author{
Lorenna M. F. Albuquerque* Orlando D. R. Filho * \\ Danúbia S. Pires* Kayon V. L. Lopes * \\ * Departamento de Eletroeletrônica, Instituto Federal do Maranhão, \\ MA, (e-mail: lorennaalbuquerque8@gmail.com; \\ orlando.rocha@ifma.edu.br; danubiapires@ifma.edu.br; \\ kayonvinicius222@hotmail.com).
}

\begin{abstract}
During the first decade of the 21st century, there was a rise in the concept of computational systems. A growing advance in the performance and flexibility of the real-time effectivity of industrial automation systems was succeeded. However, there was a need to develop sustainable projects, aiming at better management of natural resources. The main goal is that residential water management in an easy and inexpensive way promotes the consumer an awareness of the water economy in their daily life. The construction of the system is based on a fuzzy MIMO controller that was developed through a modeling and simulation software, which will lead to the control of a proportional valve, which will enable water output according to the demand of the environment and the Daily consumption report.

Resumo: Durante a primeira década do século XXI, houve uma ascensão no conceito de sistemas computacionais. Sucedeu-se então um crescente avanço no desempenho e flexibilidade da efetivação em tempo-real de sistemas de automação industrial. Contudo, existiu a necessidade de desenvolvimento de projetos sustentáveis, visando o melhor gerenciamento dos recursos naturais. O intuito principal é que o gerenciamento hídrico residencial de forma fácil e de baixo custo promova ao consumidor uma conscientização pela economia de água no seu dia a dia. A construção do sistema é baseado em um controlador MIMO nebuloso que foi desenvolvido através de um software de modelagem e simulação, o qual acarretará no controle de uma válvula proporcional, que possibilitará a saída de água conforme a demanda do ambiente e o relatório do seu consumo diário.
\end{abstract}

Keywords:

Fuzzy control; Fuzzy Logic; water flow control; proportional valve; sustainability.

Palavras-chaves: controle nebuloso; lógica nebulosa; controle de vazão hídrica; válvula proporcional; sustentabilidade.

\section{INTRODUÇÃO}

Os sistemas de controle proporcionam a uma determinada interface de entrada a solução para o seu problema específico de forma inteligente. A operação de sistemas inteligentes é associada a analogias com sistemas biológicos. A capacidade dos seres humanos em se adaptar de forma imprecisa às tomadas de decisões se diverge da possibilidade de solução das máquinas, devido ao fato de que seu raciocínio é preciso e binário. A retirada de tal restrição proporcionaria às máquinas e aos computadores a capacidade de raciocínio adaptativo, como os seres humanos. Portanto, os sistemas de controle trabalham com o processo a ser controlado e com a relação entre a entrada e saída do sistema (Shaw and Simões, 1999).

Com isso, os sistemas de controle são utilizados há muito tempo em diversas áreas, devido à sua capacidade em proporcionar uma melhor aquisição de dados e operações eficientes e, como consequência, o ganho de tempo e recursos otimizados em processos complexos ou simples (Lima and Pires, 2017). Com o aumento populacional, a sociedade vem se desenvolvendo de uma maneira que gera impactos negativos ao meio ambiente. Como consequência, o termo sustentabilidade está sendo objeto de estudo no âmbito mundial para o desenvolvimento de sistemas que utilizam os recursos naturais de maneira inteligente (Gomes and Pereira, 2018). Portanto, há um crescente ramo na comunidade científica que busca o desenvolvimento de sistemas de controle que proporcionam a otimização dos recursos naturais.

Na Europa e Ásia, diversos fabricantes adotaram a solução de controladores nebulosos em seus CLP's (Controlador Lógico Programável), empresas tais como $\mathrm{AEG}^{\mathrm{TM}}$, Siemens $\mathrm{AG}^{\mathrm{TM}}$, assim como outras. Testes foram realizados para a comprovação da eficiência de um controlador nebuloso, dentre esses, destaca-se a avaliação de um estimador de parâmetro em um sistema de freio tipo ABS que foi implementado com um bloco funcional nebuloso, o qual 
apresentou um menor tempo para o ciclo computacional se comparando a outros testes. Dessa forma, pode-se comprovar o uso do algoritmo nebuloso na maioria dos sistemas industriais (Simoes and Shaw, 2007). Com isso, a realidade do desenvolvimento de um controlador nebuloso voltado à sustentabilidade é algo exequível e está cada vez mais acessível a um grande universo de usuários, devido, também, à facilidade em aquisição de dispositivos de baixo custo (Deboni et al., 2011).

\section{PROPÓSITO}

A lógica clássica de Aristóteles fez do atributo da bivalência um marco histórico em nossa cultura ocidental. Naquela época, uma determinada informação só poderia se comportar de dois modos: falso ou verdadeiro. Não existia nada entre ambas, o meio era excluído. Entretanto, há um descompasso entre o mundo real e a nossa visão bivalente do mesmo. O mundo real é analógico, e não digital (Simoes and Shaw, 2007). Uma forma de trabalharmos os diversos graus de "verdade" é através da Lógica Nebulosa.

Os sistemas nebulosos são sistemas a serem definidos com precisão e o controlador nebuloso é um tipo especial de controle não-linear que também será definido com precisão. Embora os fenômenos que a teoria dos sistemas nebulosos caracterizam possam ser difusos, a teoria em si é precisa. Assim, torna-se eficaz o seu uso para o desenvolvimento de sistemas sem linearidade.

Observa-se que grande parte dos sistemas utilizados para controle de vazão hídrica são voltados para a eficiência. Em (Oliveira et al., 2016) é apresentada uma abordagem para otimização do uso hídrico residencial, a qual mede o consumo de água baseado nas estatísticas feitas pelo Sistema Nacional de Informações sobre Saneamento - SNIS e promove uma saída baseada nas regras de inferência Fuzzy, para que o usuário possua um melhor aproveitamento do seu recurso.

Em (Profissional et al., 2014) foi desenvolvido um protótipo de hidrômetro digital para a captação do consumo residencial de água que disponibilizou ao usuário a exibição dos dados através de gráficos em dispositivos móveis. Apesar de serem eficientes, esses sistemas não são capazes de controlar a vazão hídrica que deverá ser fornecida ao ambiente através de informações como a média do consumo diário, vazão, período e dias da semana.

O sistema MIMO inteligente nebuloso para análise e controle de vazão hídrica apresentado neste artigo tem como objetivo desenvolver um controlador nebuloso que será responsável pelo controle da vazão hídrica através da construção de uma válvula proporcional de baixo custo que proporcionará ao usuário um sistema capaz de tomar decisões de forma inteligente.

\section{MÉTODOS}

\subsection{Sistema MIMO nebuloso}

Os sistemas MIMO (múltiplas entradas e uma múltiplas saídas, do inglês, multiple input and multiple output), devem ser considerados no projeto de controle, em sistemas onde há interação de diversas entradas e várias saídas. No primeiro momento foi realizado um estudo sobre como as variáveis influenciam o sistema. A partir deste estudo, os conjuntos nebulosos foram definidos e as regras do sistema nebuloso Mamdani foram estabelecidas.

$\mathrm{Na}$ execução do sistema, foi utilizada a plataforma $A r$ duino para a aquisição de dados e operação do sistema. O Arduino que é uma plataforma de desenvolvimento de sistemas embarcados de baixo custo e open source (de Oliveira, 2017) ele foi desenvolvido para tornar mais acessível a utilização da eletrônica em projetos multidisciplinares. Sua programação é realizada em C e existem diversos modelos disponíveis no mercado (Grosskopf and Pykosz, 2017).

Para a aquisição de uma das variáveis de entrada (Vazão) foi utilizado um circuito usando dispositivos eletrônicos e o Arduino Mega. Logo após, os conjunto nebulosos da variável foram gerados. A aquisição dos dados de vazão foram obtidos através de um sensor de fluxo YF-S201 sensor que possui uma válvula em formato de catavento com um imã conectado que opera em conjunto com um sensor hall para enviar um sinal PWM. O sensor é ligado a entrada digital do Arduino, que faz o processamento do dado recebido pelo sensor. As informações são enviadas a um servidor via $W i$ - $f$, as quais foram armazenadas em um banco de dados.

O domínio da variável período é realizado através do módulo RTC Real Time Clock DS1307. Este módulo possui 56 bytes de memória não - volátil disponível para uso, arquiva e disponibiliza informações completas de data como dia da semana, dia do mês, mês, ano, horas, minutos e segundos. Este módulo também fornece o ajuste automático para meses com 31 dias e anos bissextos.

Para o construção da válvula proporcional foi utilizado um servomotor Tower Pro MG995, seu torque é apto para movimentar a haste da válvula, suas engrenagens de metal fornecem mais robustez do que engrenagens de plástico. As especificações do servomotor, estão apresentadas na tabela 1 .

Tabela 1. Especificações Servomotor Tower Pro MG995.

\begin{tabular}{cc} 
Características & Valor especificado \\
\hline Torque & $13 \mathrm{~kg} . \mathrm{cm}(4.8 \mathrm{~V})$ ou $15 \mathrm{~kg} . \mathrm{cm}(6 \mathrm{~V})$ \\
Velocidade de operação & $0,17 \mathrm{~s} / 60^{\circ}(4.8 \mathrm{~V})$ ou $0,13 \mathrm{~s} / 60^{\circ}(6 \mathrm{~V})$ \\
Tensão de operação & $4.8 \mathrm{~V}-7.2 \mathrm{~V}$ \\
Tipo de engrenagem & Engrenagem de metal \\
\hline
\end{tabular}

O registro tipo esfera com alavanca e bitola 3/8" também foi utilizado para a construção da válvula proporcional, representada na figura 1 . O registro controla a vazão que passa internamente por meio de obturador esférico. Quando a abertura do obturador e o eixo da vazão correspondem, obtém-se a vazão máxima. Do mesmo modo, quando o eixo de abertura é perpendicular à tubulação, o registro encontra-se totalmente fechado. A abertura do registro e a vazão podem ser estabelecidos muito precisamente. Este tipo de registro permite pequenas alterações na passagem de água proporcionando a possibilidade de mais estados além de totalmente aberto ou totalmente fechado, ou seja, ideal para o controle proporcional. 
Na figura 2, observam-se os componentes utilizados na montagem do circuito: (i) Arduino MEGA; (ii) Espigão Macho; (iii) Sensor de Fluxo YF-S201; (iv) módulo RTC; (v) Registro tipo esfera; (vi) Servomotor MG995; (vii) módulo ESP8266. Resistores foram utilizados para a adequação de tensão e corrente.

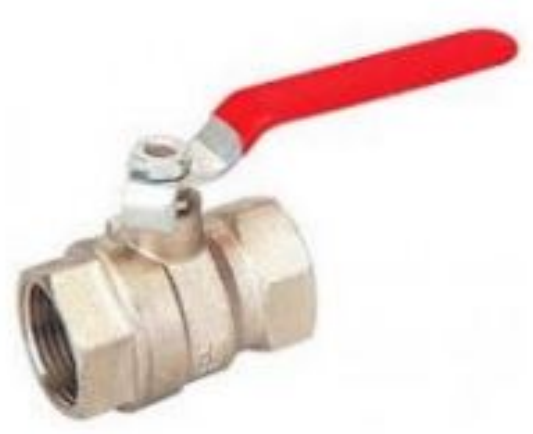

Figura 1. Registro tipo esfera 3/8". Fonte: (de Carvalho Fernandes, 2018).

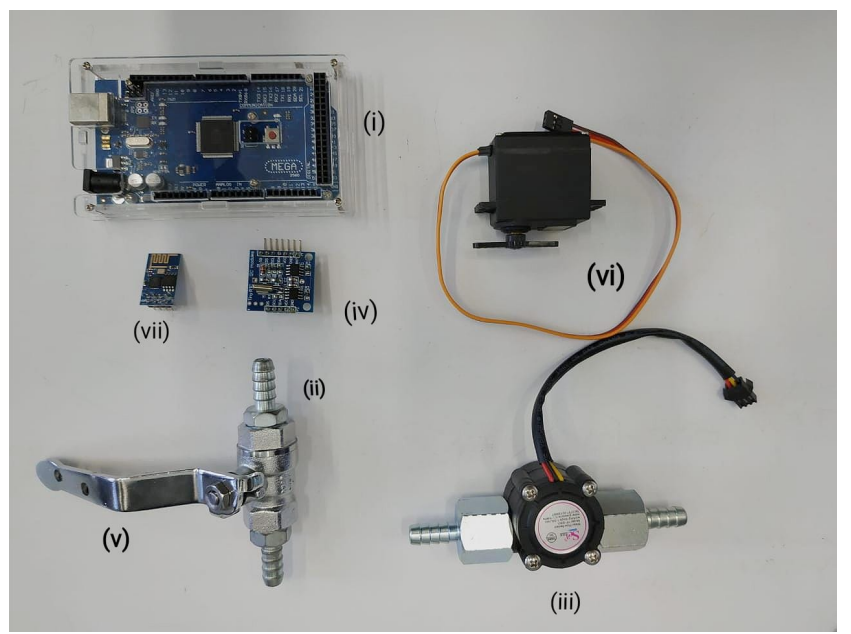

Figura 2. Componentes usados. Fonte: Autor.

\subsection{Conjuntos Nebulosos}

A vazão de água e o período do dia foram selecionadas como variáveis de entrada; realizou-se um estudo de como estas variáveis influenciam o sistema de tomada de decisão. Logo após, os conjuntos nebulosos e regras do sistema nebuloso Mamdani foram estabelecidos. A i-ésima regra do sistema nebuloso é definida a partir da expressão, dada a seguir na equação 1 .

$$
R^{(i)}: \mathrm{SE} x_{1} \mathrm{E} A_{i}^{j *} \mathrm{E} \ldots \mathrm{E} x_{n} \text { É } A_{i}^{j *} \operatorname{ENTÃO} Y \text { É } B_{n}^{m *}
$$

onde $x_{1, \ldots, n}$ corresponde às variáveis linguísticas (as variáveis definidas como entradas) do antecedente da regra nebulosa; $A_{i}^{j *}$ corresponde às partições nebulosas das variáveis linguísticas; $y$ é a saída do modelo Mamdani, representada pela partição nebulosa $B_{n}^{m *}$. O método de defuzzificação utilizado foi o do centro de gravidade ou centroide, definido pela equação 2 .

$$
\mathbf{y}^{*}=\frac{\sum_{m=1}^{N_{y}} y_{m} \mu_{0}\left(y_{m}\right)}{\sum_{m=1}^{N_{y}} y_{m}\left(\mu_{0}\right)}
$$

onde $y_{m}$ é o valor central da regra $m$ e $N_{y}$ corresponde ao número de regras do sistema nebuloso. Definida a partição do universo de discurso de cada variável em conjuntos nebulosos, foram obtidas as regras nebulosas.

Os conjuntos nebulosos para cada variável linguística do sistema podem ser observados nas figuras $3,4,5$ e 6 , respectivamente.

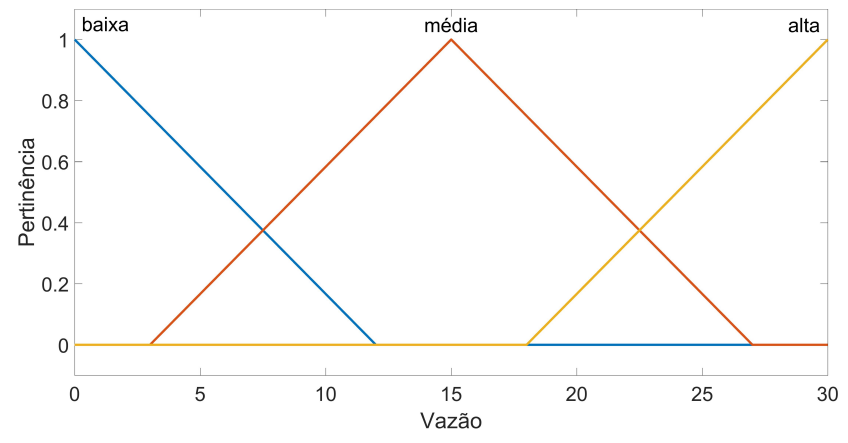

Figura 3. Funções de pertinência para variável Vazão. Fonte: Autor.

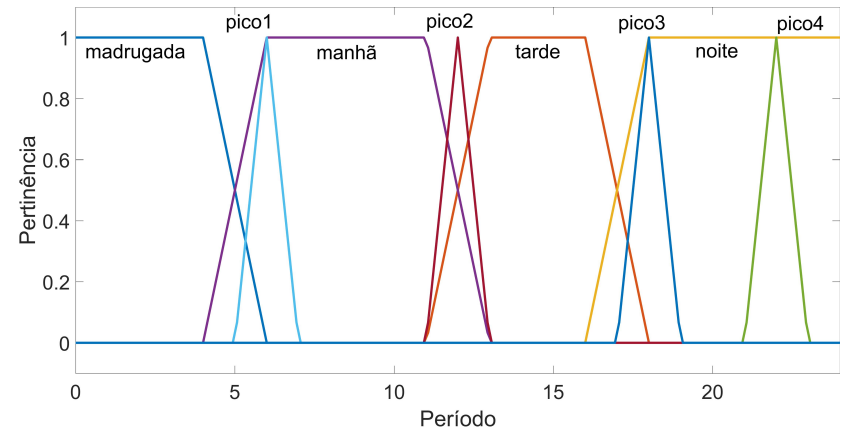

Figura 4. Funções de pertinência para variável Período. Fonte: Autor.

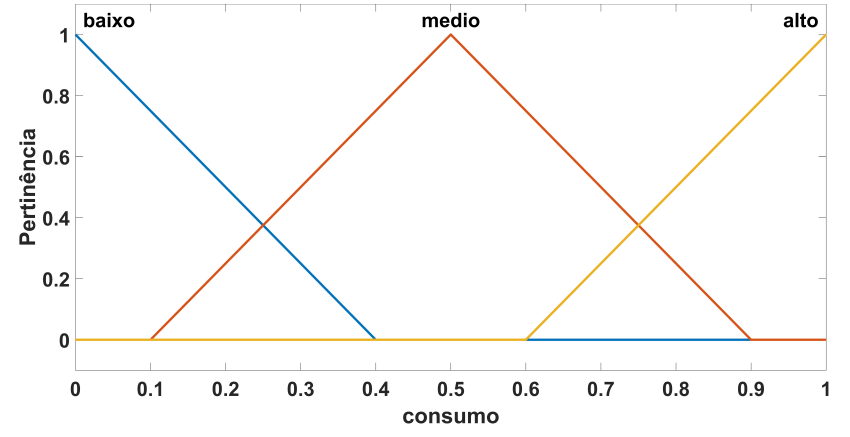

Figura 5. Funções de pertinência para variável Consumo. Fonte: Autor.

A vazão é essencial para o gerenciamento hídrico, pois por meio desta variável pode-se determinar o quantitativo hídrico utilizado em um certo período. Para a definição dos limites superior e inferior do universo de discurso 


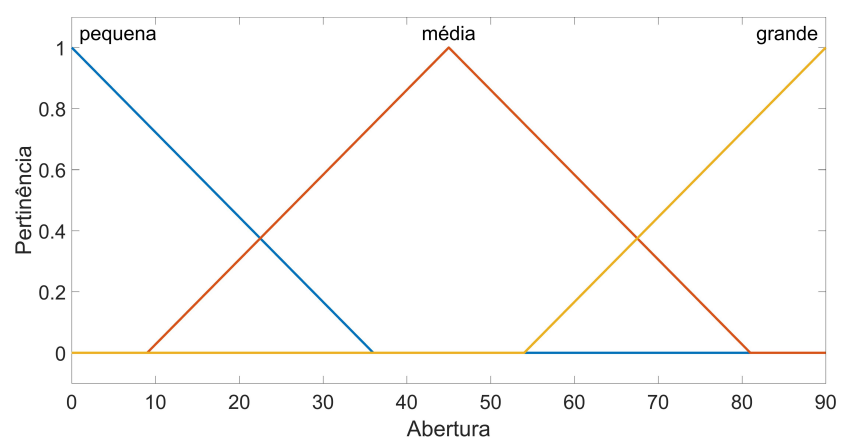

Figura 6. Funções de pertinência para variável Abertura.

Fonte: Autor.

da variável, os valores considerados, máximos e mínimos, são os valores aceitáveis para a regulação do sensor. Os conjuntos nebulosos para esta variável linguística (Figura 3) se encontram na tabela 2 .

\begin{tabular}{cc} 
Tabela 2. Variável Vazão $(\mathrm{x})$ \\
\\
Conjunto & Universo de discurso \\
\hline Baixa & $0<\mathrm{x}<12$ \\
Média & $3<\mathrm{x}<26$ \\
Alta & $18<\mathrm{x}<30$ \\
\hline
\end{tabular}

A variável Período é de suma importância em virtude de a mesma destacar os intervalos no qual o consumo se torna excessivo. Atribui-se oito conjuntos nebulosos a variável Período que é ilustrado na (Figura 4, descritos em horas do dia, conforme a tabela 3 .

\begin{tabular}{cc} 
Tabela 3. Variável Período $(\mathrm{x})$ \\
Conjunto & Universo de discurso \\
\hline Madrugada & $0<\mathrm{x}<6$ \\
Pico 1 & $5<\mathrm{x}<7$ \\
Manhã & $4<\mathrm{x}<13$ \\
Pico 2 & $11<\mathrm{x}<13$ \\
Tarde & $11<\mathrm{x}<18$ \\
Pico 3 & $17<\mathrm{x}<19$ \\
Noite & $16<\mathrm{x}<24$ \\
Pico 4 & $21<\mathrm{x}<23$ \\
\hline
\end{tabular}

Definiu-se três conjuntos para variável Consumo (Figura 5), conforme a tabela 4 .

Tabela 4. Variável Consumo(x)

\begin{tabular}{cc} 
Conjunto & Universo de discurso \\
\hline Baixo & $0<\mathrm{x}<0,4$ \\
Médio & $0,1<\mathrm{x}<0,9$ \\
Alto & $0,6<\mathrm{x}<1,0$ \\
\hline
\end{tabular}

Com a relação ao número de conjuntos nebulosos definidos para a variável de abertura da válvula, atribui-se três conjuntos nebulosos, conforme observa-se na (Figura 6, a seguir na tabela 5 , os conjuntos são descritos. A definição dos limites inferiores e superiores estão de acordo com o ângulo que o registro se movimenta.

Tabela 5. Variável Abertura da Válvula(x)

\begin{tabular}{cc} 
Conjunto & Universo de discurso \\
\hline Pequena & $0<\mathrm{x}<36$ \\
Média & $9<\mathrm{x}<81$ \\
Grande & $54<\mathrm{x}<90$ \\
\hline
\end{tabular}

\subsection{Obtenção das regras nebulosas}

Logo após a escolha das variáveis de entrada, os conjuntos nebulosos foram definidos e, por conseguinte, as regras baseadas no conhecimento prévio da aplicação do sistema. Definiram-se vinte e quatro regras que se encontram listadas na tabela 6 .

Tabela 6. Base de regras do sistema proposto

\begin{tabular}{|c|c|}
\hline Regra & Definição \\
\hline $1^{\mathrm{O}}$ & Se o período é madrugada então o consumo é baixo \\
\hline $2^{\mathrm{O}}$ & $\begin{array}{c}\text { Se o período é manhã e vazão é baixa então o consumo é } \\
\text { baixo }\end{array}$ \\
\hline $3^{\mathrm{O}}$ & $\begin{array}{c}\text { Se o período é manhã e vazão é média então o consumo } \\
\text { é médio }\end{array}$ \\
\hline$\vdots$ & $\vdots$ \\
\hline $11^{\mathrm{o}}$ & $\begin{array}{c}\text { Se o período é manhã e vazão é baixa então o abertura é } \\
\text { pequena }\end{array}$ \\
\hline $13^{\mathrm{o}}$ & $\begin{array}{c}\text { Se o período é manhã e vazão é alta então o abertura é } \\
\text { grande }\end{array}$ \\
\hline$\vdots$ & $\vdots$ \\
\hline $23^{\circ}$ & Se período é pico 3 então a abertura é grande \\
\hline $24^{\circ}$ & Se período é pico 4 então a abertura é grande \\
\hline
\end{tabular}

\section{RESULTADOS}

O controlador baseado no sistema MIMO nebuloso foi implementado na placa, em seguida realizou-se testes para verificar a aferição dos dados e a inferência do sistema. Resultados preliminares sugerem um melhor desempenho do sistema nebuloso em relação a um sistema totalmente aberto ou fechado.

Ilustra-se na Figura 4 a superfície (consumo em função da vazão e o período) obtida com a aplicação do método proposto, a partir de um modelo nebuloso Mamdani e método do centroide.

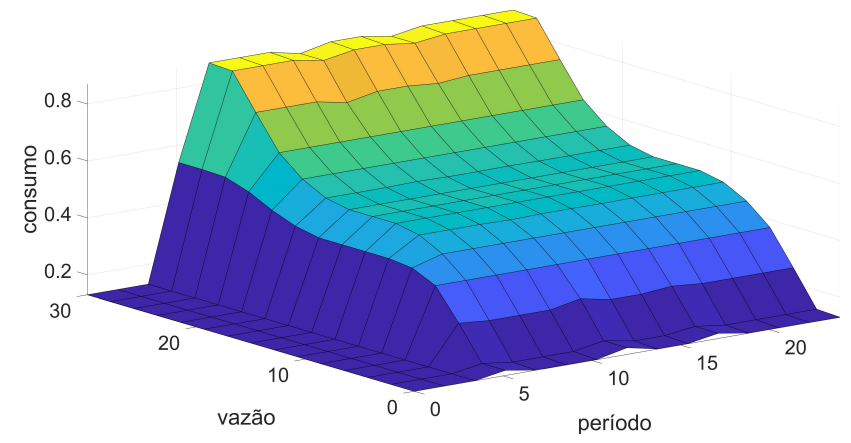

Figura 7. Curva do Consumo. Fonte: Autor.

Observa-se que o sistema demanda um consumo excessivo em períodos de pico, os quais caracterizam-se pelo período em que os consumidores encontram-se em maior quantidade utilizando a água para as necessidades pessoais e residenciais, geralmente o período de maior consumo são os períodos que os usuários estão de saída ou chegada do local de trabalho ou do seu local de estudo. Os intervalos de cada parâmetro podem mudar de acordo com o local em que o sistema pode ser empregado e suas variações máximas e mínimas de vazão. 
Ilustra-se na figura 8 a superfície (abertura da válvula em relação a vazão e período). Observa-se que o maior ângulo de abertura da válvula se encontra em períodos de pico. $\mathrm{O}$ usuário possuirá total abertura da válvula no momento de maior consumo, porém, a válvula irá se ajustar conforme a necessidade do ambiente. Desta forma, evita-se o consumo excessivo e ocasiona-se um sistema mais eficiente.

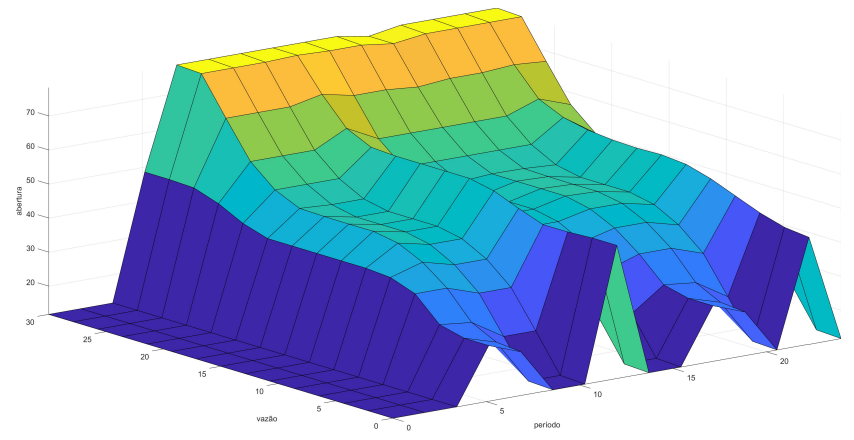

Figura 8. Curva da Abertura da Válvula. Fonte: Autor.

Ilustra-se na figura 9 o diagrama de fluxo do sistema proposto.

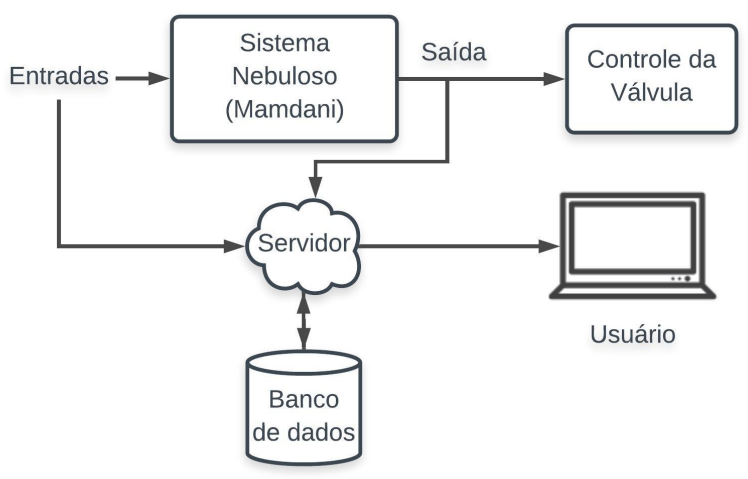

Figura 9. Fluxograma do sistema de controle. Fonte: Autor.

\section{DISCUSSÕES}

Executando alguns ajustes, pode-se obter resultados mais eficientes, uma vez trocada o método de inferência e as funções de pertinência utilizadas. As funções de pertinência escolhidas foram: triangular e trapezoidal. No princípio, a escolha se deu somente por um tipo de função, a trapezoidal. Porém, conforme os testes foram realizados, os dois tipos de funções se adequaram de uma maneira melhor. O conjunto de regras foi melhorado, estuda-se, ainda, a possibilidade de reduzir o número de regras e inserir mais uma variável de entrada. A possibilidade de usar o sensor de presença, promoverá um sistema mais preciso em relação a demanda do ambiente.

\section{CONCLUSÃO}

Uma parcela significativa dos gastos em relação ao consumo hídrico deve-se pelo uso de forma não eficiente da água. A não conscientização e o consumo não otimizado geram impactos negativos na sociedade e, consequentemente, o desperdício excessivo. Há um grande esforço científico em proporcionar meios de um desenvolvimento sustentável. Neste sentido, é exequível a produção de um sistema o qual poderá avaliar inúmeras variáveis que influenciam o ambiente, gerando assim o auxílio para tomadas de decisões eficientes. O controle e o gerenciamento de vazão através da válvula proporcional foi implementado através da plataforma Arduino para aquisição de dados e controle em tempo real. Destarte, o sistema foi executado como o esperado, armazenando os dados de entrada e saída e proporcionando ao usuário uma visão geral sobre seu consumo hídrico e o controle através da válvula proporcional.

\section{AGRADECIMENTOS}

Os autores agradecem ao Programa Institucional de Bolsas de Iniciação Científica do Instituto Federal do Maranhão (PIBIC-IFMA) e a Fundação de Amparo à Pesquisa do Estado do Maranhão (FAPEMA) pelo apoio financeiro.

\section{REFERÊNCIAS}

de Carvalho Fernandes, P. (2018). Projeto, construção e implementação de uma válvula proporcional para controle de nível em um tanque.

de Oliveira, S. (2017). Internet das coisas com ESP8266, Arduino e Raspberry PI. Novatec Editora.

Deboni, M.L., de Alvarez, C.E., and Bissoli, M. (2011). Automação sustentável: uma nova visão do emprego de tecnologias na construção civil.

Gomes, A.L.T. and Pereira, d.C.F.S. (2018). Automação residencial sustentável. REVISTA UNI-RN, 18(1/2), 29.

Grosskopf, P. and Pykosz, L.C. (2017). Controlando o consumo de ãi gua atravã (c) s da internet utilizando arduino. In Congresso Nacional de Inovação e Tecnologia.

Lima, F.S. and Pires, D.S. (2017). Sistema miso inteligente nebuloso para controle de iluminação.

Oliveira, P., Muras, A., Rodrigues, E.N.H.D., dos Santos Junior, R.d.V., Leão, F.C.P., and Lopes, D.C. (2016). Sistema inteligente baseado em regras de inferência fuzzy para eficiência hídrica doméstica.

Profissional, D.d.G.e.E., Ferreira, H.S., Heroso, L.F., and Zaleski, R.H. (2014). Sistema de monitoramento de consumo de água doméstico com a utilização de um hidrômetro digital.

Shaw, I.S. and Simões, M.G. (1999). Controle e modelagem fuzzy.

Simoes, M.G. and Shaw, I.S. (2007). Controle e modelagem fuzzy. São Paulo: Blucher: FAPESP. 